\title{
Parkinson's disease: carbidopa, nausea, and dyskinesia
}

Marty Hinz'

Alvin Stein ${ }^{2}$

Ted Cole ${ }^{3}$

'Clinical Research, NeuroResearch Clinics, Cape Coral, FL, ${ }^{2}$ Stein Orthopedic Associates, Plantation, FL, ${ }^{3}$ Cole Center for Healing, Cincinnati, OH, USA
Correspondence: Marty Hinz Clinical Research, NeuroResearch Clinics, 1008 Dolphin Drive,

Cape Coral, FL 33904, USA

Tel +l 2186262220

Fax +I 2186261638

Email marty@hinzmd.com
This article was published in the following Dove Press journal:

Clinical Pharmacology: Advances and Applications

14 November 2014

Number of times this article has been viewed

Abstract: When L-dopa use began in the early 1960s for the treatment of Parkinson's disease, nausea and reversible dyskinesias were experienced as continuing side effects. Carbidopa or benserazide was added to L-dopa in 1975 solely to control nausea. Subsequent to the increasing use of carbidopa has been the recognition of irreversible dyskinesias, which have automatically been attributed to L-dopa. The research into the etiology of these phenomena has identified the causative agent of the irreversible dyskinesias as carbidopa, not L-dopa. The mechanism of action of the carbidopa and benserazide causes irreversible binding and inactivation of vitamin $\mathrm{B}_{6}$ throughout the body. The consequences of this action are enormous, interfering with over 300 enzyme and protein functions. This has the ability to induce previously undocumented profound antihistamine dyskinesias, which have been wrongly attributed to L-dopa and may be perceived as irreversible if proper corrective action is not taken.

Keywords: vitamin B, PLP, irreversible, pyridoxal 5'-phosphate

\section{Introduction}

Serotonin, dopamine, norepinephrine, and epinephrine are centrally acting monoamines. The immediate amino acid precursor of serotonin is 5-hydroxytryptophan (5-HTP); L-3,4-dihydroxyphenylalanine (L-dopa) is the immediate amino acid precursor of dopamine. The aromatic L-amino acid decarboxylase (AADC; EC 4.1.1.28) enzyme catalyzes the synthesis of serotonin, dopamine, and histamine. ${ }^{1,2}$

Side effects may position L-dopa as one of the last drugs administered, despite the fact that it has the highest efficacy in the treatment of Parkinson's disease. ${ }^{3}$ Two prominent L-dopa side effects are nausea and dyskinesias. Carbidopa is listed as a decarboxylase inhibitor and is sold in the US. It is administered in combination with L-dopa to alleviate nausea. ${ }^{4}$ It irreversibly binds to and permanently deactivates pyridoxal 5'-phosphate (PLP) and PLP-dependent enzymes and depletes PLP reserve pools. ${ }^{5}$ The first documentation of novel carbidopa-induced dyskinesias was in $2012 .{ }^{1}$ Research into the phenomenon led to the formulation of the hypothesis that if significant depletion of histamine induces dyskinesias, then carbidopa is capable of inducing dyskinesias, which if not managed properly may be perceived as irreversible.

Benserazide is a decarboxylase inhibitor sold outside of the US. The term "benserazide" refers to the drug or its metabolite trihydroxybenzylhydrazine. No efficacy claims have been approved by the US Food and Drug Administration (FDA) for carbidopa or benserazide. ${ }^{6}$ Their only indication is management of L-dopa-induced nausea, a side effect. ${ }^{4,6}$ Double-blind studies are used to demonstrate efficacy, but are not appropriate for developing comprehensive side-effect profiles. Fatal events, which 
can occur at a rate of one in 10,000, may not be observed during a limited-population and limited-duration study.

\section{Endogenous versus competitive inhibition}

The endogenous state relating to serotonin and dopamine exists when no amino acid precursors are taken, or when inadequate or improperly balanced precursors are administered. Competitive inhibition is the interaction of serotonin and dopamine that may occur in synthesis, transport, and metabolism only when adequate and properly balanced amounts of serotonin and dopamine amino acid precursors are administered simultaneously. Organic cationtransporter type 2 functional status analysis verifies the existence of the serotonin/dopamine competitive inhibition state under the apical regulatory supersystem model. When competitive inhibition under this system exists, changes to either serotonin or dopamine concentrations individually will affect both serotonin and dopamine concentrations in a predictable manner. ${ }^{1,7-16}$

\section{Relative nutritional deficiency}

A relative nutritional deficiency (RND) exists when optimal nutrient intake cannot meet system needs. Parkinson's disease may induce many RNDs associated with depletions of serotonin, dopamine, norepinephrine, epinephrine, thiols (homocysteine, L-methionine, $S$-adenosyl-L-methionine, $S$-adenosyl-homocysteine, cystathione, L-cysteine, and glu-

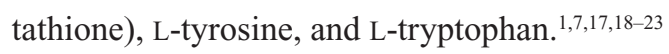

L-dopa may induce depletions of serotonin, thiols, L-tyrosine, and L-tryptophan, resulting in RNDs (Figure 1). ${ }^{1,7}$ Carbidopa may induce depletions of peripheral serotonin, dopamine, norepinephrine, and epinephrine, along with system-wide depletion of niacin and vitamin $\mathrm{B}_{6}$, resulting in multiple system RNDs. Over 300 enzymes and proteins require vitamin $\mathrm{B}_{6}$ for normal function. , $, 7,24-29^{-}$

\section{Drug/nutrient perspective}

A nutrient is any substance that facilitates normal system function. A drug is any substance that induces abnormal system function. A nutrient may become a drug. A drug may not become a nutrient. When the nutrient 5-HTP is administered as a single agent, dopamine depletion may occur. If dopamine depletion is induced, 5-HTP is no longer functioning as a nutrient; it is a drug. ${ }^{1,7-22}$ When L-dopa is administered as a single agent, it may deplete serotonin, and would then be

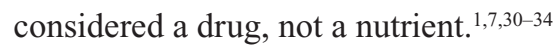

\section{L-dopa-induced nausea}

The only indication for carbidopa and benserazide is control of nausea resulting from improper L-dopa administration. The enzyme L-aromatic amino acid decarboxylase (AADC) catalyzes the synthesis of serotonin and dopamine by metabolizing 5-HTP and L-dopa, respectively. Through irreversible inhibition of AADC, carbidopa or benserazide compromises peripheral synthesis of serotonin and dopamine. This druginduced inhibition of peripheral metabolism of L-dopa by AADC leaves more L-dopa unmetabolized and available to freely cross the blood-brain barrier into the central nervous system. As a result, when carbidopa or benserazide is administered, lower L-dopa daily intake values are required to achieve the same central nervous system results., 4

It is documented that 5-HTP controls L-dopa-induced nausea, utilizing the same basic chemical mechanism as carbidopa and benserazide: AADC inhibition. Carbidopa and benserazide inhibition is irreversible while 5-HTP inhibition is reversible. The use of 5-HTP is superior, since under proper administration it is a nutrient that does not deplete systems or induce abnormal system functions when properly administered. ${ }^{1,7,35-37}$

If the goal of administering 5-HTP for the control of L-dopa-induced nausea is to have it function as a nutrient, this is not merely a simple substitution. It requires concomitant

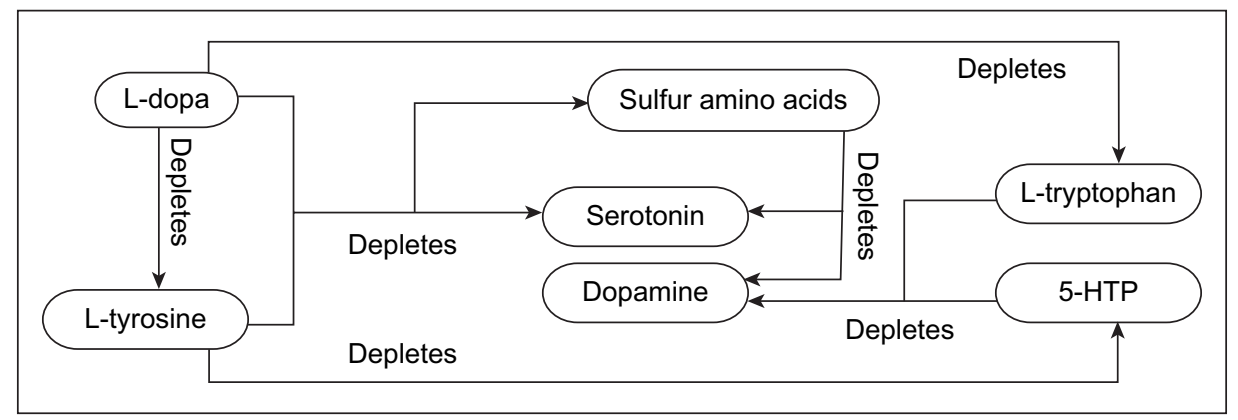

Figure I Administering any of the illustrated components in a dominant manner will facilitate the associated depletion.

Notes: Copyright (C) 2012. Dove Medical Press. Adapted from Hinz M, Stein A, Uncini T. The discrediting of the monoamine hypothesis. Int J Gen Med. 20I2;5:I35-142.15 Abbreviation: HTP, hydroxytryptophan. 
administration of L-dopa with 5-HTP, along with the "core nutrients": L-tyrosine, a thiol (L-cysteine, glutathione, $S$-adenosyl methionine, or L-methionine), and cofactors (vitamin C, vitamin $\mathrm{B}_{6}$, and calcium carbonate). Administration of properly balanced core nutrients needs to be guided by organic cation-transporter type 2 functional status analysis, in order to achieve a balance that does not convert the nutrients into a drug. ${ }^{1,737}$

All L-dopa single-ingredient products were discontinued by the pharmaceutical companies in the US in $1999 .{ }^{38}$ Concomitant L-dopa/carbidopa products became the only prescription form of L-dopa available. If an indication for L-dopa as a single ingredient is identified, it is available only as a nutritionally sourced product, such as Mucuna cochinchinensis (which is defined by the US Department of Agriculture as a synonym for Mucuna pruriens). ${ }^{38,39}$ Few physicians are aware of the existence of this product.

\section{Carbidopa dyskinesias}

Abnormal or impaired voluntary movements are dyskinesias. ${ }^{40}$ Movement disorders associated with Parkinson's disease include tremors, and other uncoordinated motions. These are often seen when there are inadequate levels of L-dopa available in the system. For over 50 years, all dyskinesias experienced while taking L-dopa or concomitant carbidopa/ L-dopa preparations were described as L-dopa-induced dyskinesias. ${ }^{41-48}$ It is common for dyskinesia studies that reference administration of concomitant L-dopa/carbidopa to refer to the combination as L-dopa. ${ }^{49}$

Novel observations reporting a group of 17 Parkinson's disease patients led to identification of carbidopa-induced dyskinesias, which had not been documented prior to $2012 .{ }^{1}$ These patients had been ingesting prescribed concomitant L-dopa/carbidopa preparations for 1-7 years. Their drugs were continued as the core nutrients were started. The mean daily dosing value of L-dopa/carbidopa was 1,000 $\mathrm{mg}$ and $250 \mathrm{mg}$, respectively, at the initiation of the core nutrients. The mean duration of previous drug treatment was 3 years, 7 months. Onset of dyskinesias began within the first week of treatment when the core nutrients were added. Dyskinesias were generally described as facial twitching and head bobbing due to peripheral muscle-control problems within the neck and upper shoulders. When dyskinesias developed, the L-dopa/ carbidopa was immediately discontinued and the nutritionally sourced L-dopa increased fivefold to compensate for the loss of the carbidopa effect on the central nervous system by blocking peripheral conversion of L-dopa to dopamine. This conversion to higher-dose L-dopa was monitored using the previously published pill-stop technique. ${ }^{37}$ Following this protocol, all patients achieved full resolution of dyskinesias within 4 days. This led to the hypothesis that the dyskinesias that resolved after applying the new protocol had been induced by the carbidopa and were not related to the L-dopa. There were no refractory dyskinesias experienced.

\section{PLP}

Histidine decarboxylase (HDC) and AADC are PLP-dependent enzymes that catalyze the metabolism of histidine to histamine. ${ }^{50}$ Each contains an apoprotein core requiring irreversible hydrazine-PLP binding for activation. PLP then becomes the active site for catalyzing reactions. ${ }^{5}$ Carbidopa and benserazide induce permanent deactivation and irreversible binding of PLP, PLP-dependent enzymes, and depletion of PLP reserve pools. ${ }^{1,24}$ While PLP freely crosses the bloodbrain barrier, ${ }^{51}$ carbidopa and benserazide do not. ${ }^{4,6}$ If carbidopa and benserazide deplete peripheral PLP, then central PLP is also depleted when the system reaches equilibrium. Over 300 enzymes and proteins may be adversely affected by this extensive PLP collapse. ${ }^{24}$

\section{Semantic change and neologisms}

Dyskinesias are classified as either reversible or irreversible. Between 1960 and 1975, when only the single-ingredient L-dopa was available, no L-dopa-induced irreversible dyskinesias were documented. ${ }^{52}$ In 1975, the FDA approved the combined formulation of L-dopa with carbidopa for Parkinson's disease treatment. The neologism "L-dopainduced irreversible dyskinesias" first appeared in the literature after $1975 .{ }^{53}$ A semantic change had occurred: the term "L-dopa-induced dyskinesias" was expanded to include L-dopa-induced irreversible dyskinesias.

A 1971 article noted: "Transitory adventitious movements are the commonest dose-limiting adverse reaction to levodopa in parkinsonian patients. Prompt resolution of dyskinetic symptoms usually attends reduction in levodopa dosage or the administration of pyridoxine hydrochloride." 54 A 1999 article noted: "As Parkinson's disease progresses, the dosage and frequency of levodopa needs to be increased to maintain control. As a result, most patients develop irreversible dyskinesias." ${ }^{55}$ A 2012 article noted: "The most effective pharmacologic treatment for PD, levodopa, has a limited period of effectiveness (7-12 years) and is associated with irreversible dyskinesias." 56

\section{Antihistamine dyskinesias}

To formulate the following observations, Hinz et al treated over 800 Parkinson's disease patients primarily with Mucuna 
pruriens-sourced L-dopa, with and without carbidopa. An antihistamine is a substance that inhibits the histamine agonist, inhibits histamine synthesis, or induces physiologic antihistamine effects. ${ }^{57}$ Antihistamine effects may be exerted by 5-HTP (weak), L-dopa (strong), and carbidopa (potentially profound). AADC or HDC is exclusively responsible for catalyzing histamine synthesis. If 5-HTP induces a reversible inhibition of AADC, then a weak antihistamine effect may be present. ${ }^{1,16}$ This is a novel documentation of 5-HTP having a potential antihistamine effect.

L-dopa may induce both strong and weak antihistamine effects. Dopamine metabolism to epinephrine is without biochemical regulation. Synthesis of epinephrine shares a direct relationship with dopamine. A strong physiologic antihistamine effect is associated with increased epinephrine synthesis induced by administration of L-dopa. The rapid power of epinephrine to reverse acute life-threatening histamine collapse is well known in medicine. ${ }^{57} \mathrm{~L}$-dopa also induces a second weaker antihistamine effect through reversible inhibition of AADC. ${ }^{1}$

Carbidopa and benserazide have the ability to induce a previously undocumented profound antihistamine effect. These drugs irreversibly bind to and permanently deactivate the two PLP-dependent enzymes responsible for histamine synthesis: AADC and HDC. ${ }^{5,58,59}$ This is exacerbated by depletion of their PLP substrate and PLP reserve pools. ${ }^{5}$ Dyskinesias may be induced by prolonged high-dose antihistamine ingestion or overdose. ${ }^{60-63}$ If carbidopa is administered in high-enough and/or long-enough concentrations to cause significant collapse of histamine synthesis, then carbidopainduced antihistamine dyskinesias will occur.

\section{Discussion}

L-dopa-induced dyskinesias have not been observed or documented in the serotonin/dopamine competitive inhibition state. Prior to 1976 and the introduction of carbidopa, there was no documentation of "L-dopa-induced irreversible dyskinesias." 52

The molecular weight of PLP is $247.142 .{ }^{63}$ Carbidopa has a molecular weight of 244.244. ${ }^{64}$ Molecular binding of carbidopa to PLP is in a 1:1 ratio. Free adult male PLP is placed at $167 \mathrm{mg} .{ }^{65}$ The PLP that is noncovalently bound to about 300 enzymes and proteins represents the PLP reserve pool, which is at equilibrium with free PLP. ${ }^{24}$ The amount of PLP bound and unbound system-wide is not agreed upon. Assume carbidopa ingestion of $250 \mathrm{mg}$ per day over a 4-year period, while ingesting the US-recommended daily allowance of about $2 \mathrm{mg}$ of vitamin $\mathrm{B}_{6}$ per day. This reveals a potential irreversible carbidopa PLP-induced deficiency of $0.36208 \mathrm{~kg}$ $(0.78 \mathrm{lb})$. If PLP depletion is great enough, then administration of $2 \mathrm{mg}$ per day of vitamin $\mathrm{B}_{6}$ will never reverse the profound drug-induced PLP RND and the carbidopa-induced antihistamine dyskinesias in the patient's lifetime. The observed dyskinesias will be perceived as irreversible.

Effective control of reversible L-dopa-induced dyskinesias with PLP was documented in the 15-year precarbidopa era. ${ }^{54}$ It is documented that administration of adequate amounts of PLP will reverse the AADC and HDC effects from carbidopa when it is stopped. ${ }^{66}$ If discontinuation of carbidopa with administration of ample vitamin $B_{6}$ is effective over time at reversing the effects of irreversible binding of carbidopa to AADC and HDC as new molecules of these enzymes are synthesized, then the approach will be effective in reversing carbidopa-induced antihistamine dyskinesias. The PLP reserve pool is much larger than previously realized. It is made up of about 300 enzymes and proteins that are noncovalently bound to PLP. If the PLP reserve pool is significantly greater than anticipated, then adequate reversal of carbidopa PLP collapse may take administration of PLP-dosing values in excess of those previously associated with restoration of PLP collapse. For optimal restoration of PLP collapse, carbidopa or benserazide needs to be discontinued.

\section{Conclusion}

This paper discusses the mechanism of action whereby 5-HTP controls L-dopa-induced nausea. It also discusses the previously undocumented potential of carbidopa and benserazide to induce profound antihistamine dyskinesias that are irreversible if ample vitamin $\mathrm{B}_{6}$ replacement is not provided.

During the 15 years prior to 1975 , when L-dopa was prescribed without carbidopa, there was no documentation of irreversible dyskinesias. After the FDA approval of carbidopa/L-dopa, the neologism "L-dopa-induced irreversible dyskinesias" began to appear in the literature as a semantic change. After carbidopa was approved by the FDA in 1975 for concomitant administration with L-dopa, all new-onset dyskinesias have continued to be wrongly attributed to L-dopa alone. Documentation of carbidopa-induced dyskinesias did not exist until 2012. Irreversible antihistamine dyskinesias are a function of carbidopa's mechanism of action, which causes collapse of histamine synthesis that is exacerbated by PLP collapse and PLP reserve-pool depletion.

The possible hesitation of the medical community to embrace the nutritional approach by giving up the drugs (carbidopa or benserazide) is without foundation. 
The efficacy of L-dopa without the drugs carbidopa or benserazide has been amply documented in double-blind studies since 1960. There are no efficacy claims for these drugs approved by the FDA. Properly administering the nondrug protocol that incorporates the core nutrients definitively controls L-dopa-induced nausea and avoids irreversible dyskinesias. No carbidopa or benserazide is utilized in this regimen, thereby eliminating the massive vitamin $\mathrm{B}_{6}$ depletion experienced by patients taking the combined L-dopa/ carbidopa formulation. This nutritional approach should raise no concern, as all of the ingredients are classified by the FDA as being safe enough for over-the-counter sales.

\section{Disclosure}

MH discloses his relationship with CHK Nutrition, which was wholly divested in June 2011, along with current affiliations with DBS Labs and NeuroResearch Clinics. The other authors report no conflicts of interest in this work.

\section{References}

1. Hinz M, Stein A, Uncini T. Relative nutritional deficiencies associated with centrally acting monoamines. Int J Gen Med. 2012;5:413-430.

2. GenomeNet. Homo sapiens (human): 1644. Available from: http:// www.genome.jp/dbget-bin/www_bget?hsa:1644+H01161+D00405+ D00558+D01653+D03082+D08205. Accessed August 2, 2014.

3. Mayo Clinic. Parkinson's disease: treatments and drugs. 2014. Available from: http://www.mayoclinic.org/diseases-conditions/parkinsonsdisease/basics/treatment/con-20028488. Accessed August 2, 2014.

4. Sinemet $\mathrm{CR}^{\circledR}$ [prescribing information]. Available from: http:// packageinserts.bms.com/pi/pi_sinemet_cr.pdf. Accessed August 2, 2014.

5. Daidone F, Montioli R, Paiardini A, et al. Identification by virtual screening and in vitro testing of human DOPA decarboxylase inhibitors. PLoS One. 2012;7(2):e31610.

6. Roche Australia. Madopar ${ }^{(B}$ [prescribing information]. Sydney: Roche Australia; 2010. Available from: http://www.roche-australia.com/ content/dam/internet/corporate/roche/en_AU/files/central_nervous_ agents/madopar-pi.pdf. Accessed August 2, 2014.

7. Hinz M, Stein A, Uncini T. Amino acid management of Parkinson disease: a case study. Int J Gen Med. 2011;4:1-10.

8. Hinz M, Stein A, Uncini T. Validity of urinary monoamine assay sales under the "spot baseline urinary neurotransmitter testing marketing model". Int J Nephrol Renovasc Dis. 2011;4:101-113.

9. Hinz M, Stein A, Uncini T. APRESS: apical regulatory super system, serotonin, and dopamine interaction. Neuropsychiatr Dis Treat. 2011;2011:7 1-7.

10. Hinz M, Stein A, Uncini T. The dual-gate lumen model of renal monoamine transport. Neuropsychiatr Dis Treat. 2010;6:387-392.

11. Stein A, Hinz M, Uncini T. Amino acid responsive Crohn's disease: a case study. Clin Exp Gastroenterol. 2010;3:171-177.

12. Hinz M, Stein A, Uncini T. Treatment of attention deficit hyperactivity disorder with monoamine amino acid precursors and organic cation transporter assay interpretation. Neuropsychiatr Dis Treat. 2011;7: 31-38.

13. Hinz M, Stein A, Uncini T. Urinary neurotransmitter testing: considerations of spot baseline norepinephrine and epinephrine. Open Access J Urol. 2011;3:19-24.

14. Hinz M, Stein A, Uncini T. Monoamine depletion by reuptake inhibitors. Drug Healthc Patient Saf. 2011;3:69-77.
15. Hinz M, Stein A, Uncini T. The discrediting of the monoamine hypothesis. Int J Gen Med. 2012;5:135-142.

16. Hinz M, Stein A, Uncini T. 5-HTP efficacy and contraindications. Int J Gen Med. 2012;5:413-430.

17. Carta M, Carlsson T, Muñoz A, Kirik D, Björklund A. Serotonindopamine interaction in the induction and maintenance of L-DOPAinduced dyskinesias. Prog Brain Res. 2008;172:465-468.

18. Mones RJ, Elizan TS, Siegel GJ. Analysis of L-dopa induced dyskinesias in 51 patients with parkinsonism. J Neurol Neurosurg Psychiatry. 1971;34:668-673.

19. Chase TN. Serotonergic mechanisms in Parkinson's disease. Arch Neurol. 1972;27:354-356.

20. Busch AE, Karbach U, Miska D, et al. Human neurons express the polyspecific cation transporter hOCT2, which translocates monoamine neurotransmitters, amantadine, and memantine. Mol Pharmacol. 1998;54:342-352.

21. Mayeux R, Stern Y, Williams JB, Cote L, Frantz A, Dyrenfurth I. Clinical and biochemical features of depression in Parkinson's disease. Am J Psychiatry. 1986;143:756-759.

22. Chan-Palay V, Höchli M, Jentsch B, Leonard B, Zetsche T. Raphe serotonin neurons in the human brain stem in normal controls and patients with senile dementia of the Alzheimer type and Parkinson's disease: relation to monoamine oxidase enzyme location. Dementia. 1992;3:253-269.

23. Charlton CG, Mack J. Substantia nigra degeneration and tyrosine hydroxylase depletion caused by excess S-adenosylmethionine in the rat brain: support for an excess methylation hypothesis for parkinsonism. Mol Neurobiol. 1994;9:149-161.

24. UniProt. Search query results. Available from: http://www.uniprot.org/ uniprot/?query=pyridoxal+AND+organism $\% 3 \mathrm{~A} \% 22$ Homo+sapiens+\% 5B9606\%5D\%22\&sort=score. Accessed July 4, 2014.

25. Andrews DW, Patrick RL, Barchas JD. The effects of 5-hydroxytryptophan and 5-hydroxytryptamine on dopamine synthesis and release in rat brain striatal synaptosomes. J Neurochem. 1978;30:465-470.

26. Awazi N, Guldberg HC. On the interaction of 5-hydroxytryptophan and 5-hydroxytryptamine with dopamine metabolism in the rat striatum arch. Naunyn Schmiedebergs Arch Pharmacol. 1978;303:63-72.

27. Zhelyaskov DK, Levitt M, Udenfriend S. Tryptophan derivatives as inhibitors of tyrosine hydroxylase in vivo and in vitro. Mol Pharmacol. 1968;4:445-451.

28. Ng LK, Chase TN, Colburn RW, Kopin IJ. Research of $\left[{ }^{3} \mathrm{H}\right]$ dopamine by L-5-hydroxytryptophan. Brain Res. 1972;45:499-505.

29. Stamford JA, Kruk ZL, Millar J. Striatal dopamine terminals release serotonin after 5-HTP pretreatment: in vivo voltammetric data. Brain Res. 1990;515:173-180.

30. Ritvo ER, Yuwiler A, Geller E, et al. Effects of L-dopa in autism. J Autism Child Schizophr. 1971;1:190-205.

31. Wuerthele SM, Moore KE. Studies on the mechanisms of L-dopa induced depletion of 5-hydroxytryptamine in the mouse brain. Life Sci. 1977;20:1675-1680.

32. Borah A, Mohanakumar KP. Long-term L-DOPA treatment causes indiscriminate increase in dopamine levels at the cost of serotonin synthesis in discrete brain regions of rats. Cell Mol Neurobiol. 2007;27: 985-996.

33. Karobath M, Díaz JL, Huttunen MO. The effect of L-dopa on the concentrations of tryptophan, tyrosine, and serotonin in rat brain. Eur J Pharmacol. 1971;14:393-396.

34. García NH, Berndt TJ, Tyce GM, Knox FG. Chronic oral L-DOPA increases dopamine and decreases serotonin excretions. Am J Physiol. 1999;277:R1476-R1480.

35. Carta M, Carlsson T, Kirik D, Björklund A. Dopamine released from 5-HT terminals is the cause of L-DOPA-induced dyskinesia in parkinsonian rats. Brain. 2007;130:1819-1833.

36. Everett GM, Borcherding JW. L-DOPA: effect on concentrations of dopamine, norepinephrine, and serotonin in brains of mice. Science. 1970;168:847-850. 
37. Hinz M, Stein A, Cole T. Management of L-dopa overdose in the competitive inhibition state. Drug Healthc Patient Saf. 2014;6: 93-99.

38. US Department of Agriculture. Mucuna chochinchinensis (Lour) A Chev Available from: http://www.ars-grin.gov/cgi-bin/npgs/html/ taxon.pl?70451. Accessed August 3, 2014.

39. US Food and Drug Administration. FDA approved drug products. Available from: http://www.accessdata.fda.gov/scripts/cder/drugsatfda/ index.cfm. Accessed September 22, 2014.

40. Google. Dyskinesia definition. Available from: https://www.google. $\mathrm{com} /$ search?sourceid=navclient\&ie=UTF-8\&rlz=1T4GGHP_enUS59 0US590\&q=dyskinesia+definition. Accessed August 2, 2014.

41. Barbeau A. L-dopa therapy in Parkinson's disease: a critical review of nine years' experience. Canada Med Assoc J. 1969;101:59-68.

42. Krack P, Pollak P, Limousin P, et al. Subthalamic nucleus or internal pallidal stimulation in young onset Parkinson's disease. Brain. 1998;121: 451-457.

43. Fabbrini G, Brotchie JM, Grandas F, Nomoto M, Goetz CG. Levodopainduced dyskinesias. Mov Disord. 2007;22:1379-1389; quiz 1523.

44. Caparros-Lefebvre D, Blond S, Vermersch P, Pécheux N, Guieu JD, Petit H. Chronic thalamic stimulation improves tremor and levodopa induced dyskinesias in Parkinson's disease. J Neurol Neurosurg Psychiatry. 1993;56:268-273.

45. Jankovic J. Parkinson's disease: clinical features and diagnosis. J Neurol Neurosurg Psychiatry. 2008;79:368-376.

46. Fahn S. Levodopa in the treatment of Parkinson's disease. J Neural Transm Suppl. 2006:1-15.

47. Del Sorbo F, Albanese A. Levodopa-induced dyskinesias and their management. J Neurol. 2008;255 Suppl 4:32-41.

48. Lewitt PA, Hauser RA, Lu M, et al. Randomized clinical trial of fipamezole for dyskinesia in Parkinson disease (FJORD study). Neurology. 2012;79:164-169.

49. Rascol O, Brooks DJ, Korczyn AD, De Deyn PP, Clarke CE, Lang AE. A five-year study of the incidence of dyskinesia in patients with early Parkinson's disease who were treated with ropinirole or levodopa. N Engl J Med. 2000;342;1484-1491.

50. Paiardini A, Contestabile R, Buckle AM, Cellini B. PLP-dependent enzymes. Biomed Res Int. 2014;2014;856076.

51. Giardinia G, Montioli R, Gianni S, et al. Open conformation of human DOPA decarboxylase reveals the mechanism of PLP addition to group II decarboxylases. Proc Natl Acad Sci U S A. 2011;108: 20514-20519.
52. Google Scholar. Search: [parkinson's irreversible dyskinesias "l-dopa or levodopa"] with query prior to 1977. Accessed August 2, 2014.

53. Schreck J, Kelsberg G, Rich J, Ward R. Clinical inquiries. What is the best initial treatment of Parkinson's disease? J Fam Pract. 2003;52: 897-899.

54. Weiss JL, Ng LK, Chase TN. Long-lasting dyskinesia induced by L-dopa. Lancet. 1971;1:1016-1017.

55. Greener M. New Hope for patients with Parkinson's disease. Inpharma. 1999:3-4.

56. Landers M. Exercise-Induced Neuroprotection in a Hemiparkinsonian 6-Hydroxydopamine Rat Model [doctoral thesis]. Las Vegas: University of Nevada; 2012.

57. Oxford Dictionaries. Definition of antihistamine. Available from: http:// www.oxforddictionaries.com/us/definition/american_english/antihistamine. Accessed August 2, 2014.

58. Kemp SF, Lockey RF, Simons FE. Epinephrine: the drug of choice for anaphylaxis. A statement of the World Allergy Organization. Allergy. 2008;63:1061-1070.

59. Wu F, Christen P, Gehring H. A novel approach to inhibit intracellular vitamin B6-dependent enzymes: proof with human and plasmodium ornithine decarboxylase and human histidine decarboxylase. FASEB J. 2011;25:2109-2122.

60. Olson K. Poisoning and Drug Overdose. 4th ed. New York: McGrawHill1; 2003.

61. Edriss H, Pfarr M. Acute respiratory distress syndrome, metabolic acidosis, and respiratory acidosis associated with citalopram overdose. 2014. Available from: http://pulmonarychronicles.com/ojs/index.php?jo urnal $=$ pulmonarychronicles $\&$ page $=$ article $\& o p=$ view $\&$ path $\% 5 \mathrm{~B} \% 5 \mathrm{D}=$ 102\&path\%5B\%5D=213. Accessed August 2, 2014.

62. Scharman EJ1, Erdman AR, Wax PM, et al. Diphenhydramine and dimenhydrinate poisoning: an evidence-based consensus guideline for out-of-hospital management. Clin Toxicol (Phila). 2006;44:205-223.

63. DrugBank. Pyridoxal phosphate. Available from: http://www.drugbank. ca/drugs/DB00114. Accessed July 20, 2014.

64. DrugBank. Carbidopa. Available from: http://www.drugbank.ca/drugs/ DB00190. Accessed July 20, 2014

65. Pitkin R. Dietary Reference Intakes for Thiamin, Riboflavin, Niacin, Vitamin $B_{6}$, Folate, Vitamin $B_{12}$, Pantothenic Acid, Biotin, and Choline. Washington: National Academies; 1998.

66. Hinz M, Stein A, Cole T. Parkinson's disease death rate: carbidopa and vitamin B6 (PLP). Clinical Pharmacology: Advances and Applications. 2014;6:161-169.
Clinical Pharmacology: Advances and Applications

\section{Publish your work in this journal}

Clinical Pharmacology: Advances and Applications is an international, peer-reviewed, open access journal publishing original research, reports, reviews and commentaries on all areas of drug experience in humans. The manuscript management system is completely online and includes a very quick and fair peer-review system, which is all easy to use.

\section{Dovepress}

Visit http://www.dovepress.com/testimonials.php to read real quotes from published authors. 